\title{
Secure Hybrid RF/VLC under Statistical Queuing Constraints
}

\author{
I. W. Gomes da Silva*, D. P. Moya Osorio ${ }^{\dagger}$, E. E. Benitez Olivo ${ }^{\ddagger}$, I. Ahmed ${ }^{\dagger}$ and M. Katz \\ *Department of Electrical Engineering, Federal University of São Carlos, São Carlos, Brazil \\ ${ }^{\dagger} 6 \mathrm{G}$ Flagship, University of Oulu, Finland \\ ${ }_{\text {† }}$ ão Paulo State University (UNESP), Campus of São João da Boa Vista, Brazil \\ E-mails: isabella@estudante.ufscar.br, diana.moyaosorio@oulu.fi, edgar.olivo@unesp.br,iqrar.ahmed@oulu.fi, marcos.katz@oulu.fi
}

\begin{abstract}
This paper evaluates the effective capacity and maximum average arrival rate of a hybrid radio frequency (RF) and visible light communication (VLC) network in the presence of an eavesdropper. It is assumed that the data is first stored in a buffer prior to transmission. Thus, it is considered that the source operates under constraints of buffer overflow probabilities. We also consider that the data is transmitted over the RF and VLC links following a proposed multiplex scheme in which the buffer service rate is described in terms of the secrecy capacity and split with a certain allocation ratio. Moreover, the legitimate and eavesdropper users are assumed to have multihoming capabilities so that are able to receive data from both access points simultaneously. We formulate an integral-form and asymptotic expressions for the effective capacity and validate them via Monte Carlo simulations. From the numerical results, we show that combining RF and VLC and splitting the data among them can enhance the performance in terms of the buffer quality of service $(\mathrm{QoS})$ constraints and secrecy requirements.

Index Terms-Effective capacity, hybrid RF/VLC, physical layer security, visible light communications.
\end{abstract}

\section{INTRODUCTION}

The fifth-generation of wireless communications (5G) represents a significant leap forward compared with previous generations. However, as modern society heavily relies on wireless communications and adapts to the presence and availability of $5 \mathrm{G}$ systems, new challenges are expected with the appearance of novel use cases. By 2030, the future sixthgeneration $(6 \mathrm{G})$ is expected to have an even more pronounced and integrated role in digital societies, by offering virtually zero-latency services, unlimited storage, and immense cognition capabilities [1]. Moreover, to achieve even greater data rates, $6 \mathrm{G}$ will have to exploit different and innovative enabling technologies.

In $6 \mathrm{G}$, it will be required to go beyond $100 \mathrm{GHz}$ in order to alleviate the scarcity of spectrum. With an unlicensed frequency range of $400-800 \mathrm{THz}$, visible-light communication (VLC) has emerged as one of the most promising technologies and has attracted considerable interest motivated by the advance in white light-emitting diodes (LEDs) and its unique advantages such as the capability of providing illumination and data transmission simultaneously, high energy efficiency, and longer lifespan. Moreover, other interesting features are the inherent security and privacy, reduced costs, and high-speed transmissions [2], [3]. However, in spite of the encouraging characteristics and advantages of VLC systems, numerous challenges and limitations must be addressed for the deployment of VLC in practical scenarios. Among them, we can cite the limited coverage and instability of the link quality [4]. To overcome these limitations, several works in the literature have proposed hybrid solutions by combining VLC and radio frequency (RF) communications [2], [5]. In [2], the authors presented, through an experimental setup, a reconfigurable optical and RF wireless system, which is capable of dynamically adopting the best mode for transmission based on predefined handover rules, e.g., link failures or local policies. Besides, the authors in [5] presented a comparison between hybrid RF/VLC systems and a stand-alone VLC network. Therein, it was shown a remarkable gain in terms of average connectivity and system throughput.

On the other hand, the assurance of security and privacy is undoubtedly a key requirement for future $6 \mathrm{G}$ networks, where traditional cryptography-based techniques might be unsuitable or insufficient for different applications [6]. In this sense, physical layer security (PLS) techniques have shown to be a promising alternative for providing security by efficiently exploiting the wireless medium's properties, to provide a further level of protection. A few works with the co-deployment of RF and VLC have investigated networks under secrecy constraints and PLS techniques. For example, in [7] and [8], the authors propose PLS algorithms based on zero-forcing beamforming to prevent the eavesdropper from receiving data from both VLC and RF.

To the best of the author's knowledge, security issues for hybrid RF/VLC systems have been barely investigated. In this paper, we intend to fill this gap by considering a hybrid RF/VLC network in the presence of an eavesdropper with a buffer-aided source that operates under statistical QoS constraints. Notably, the main contributions of the paper are as follows: i) To take advantage of the best characteristics of the RF and VLC domains, we propose a multiplexing scheme where the service rate, described by the secrecy capacity is split according to a certain allocation ratio; ii) An integralform expression for the effective capacity is derived to assess key system parameters' effect on the network performance; iii) An asymptotic expression of the effective capacity is obtained. Moreover, both expressions are validated via Monte Carlo simulations.

Notation: Herein we use $f_{X}(\cdot)$ and $F_{X}(\cdot)$ to denote 
the probability density function (PDF) and cumulative density function $(\mathrm{CDF})$ of a random variable $X ; \mathbb{E}[\cdot]$ to denote expectation; $I_{0}(\cdot)$ and $\Gamma(\cdot)$ stands for the zeroorder modified Bessel function of the first kind [9, Eq. 8.447.1] and the gamma function [9, Eq. 8.324], respectively; ${ }_{2} F_{1}(\cdot ; \cdot ; \cdot)$ is the hypergeometric function [9, Eq. 9.14.2]; and $[x]^{+}=\max (x, 0)$.

\section{SySTEM MODEL}

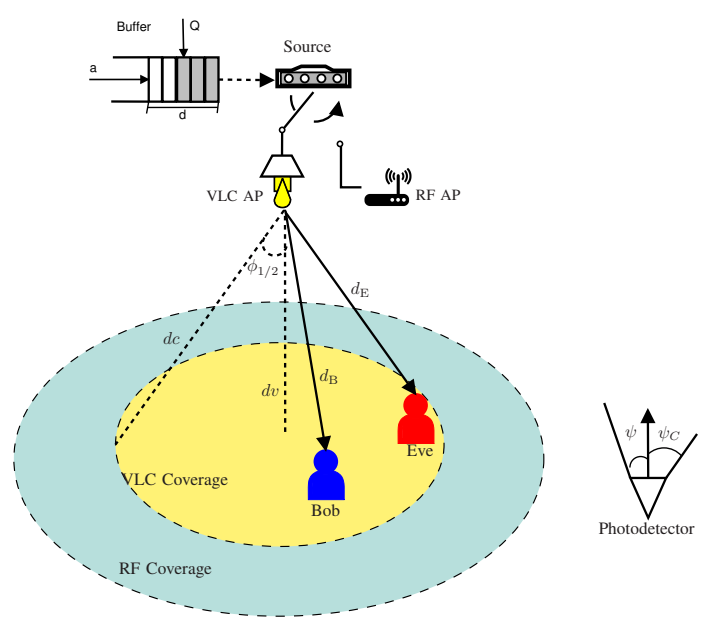

Fig. 1: System Model

Fig. 1 illustrates an indoor downlink hybrid VLC/RF network where a source (S) intends to communicate with a destination (Bob) in the presence of an eavesdropper (Eve), through either RF or VLC access points (APs). We assume that $\mathrm{S}$ receives data from the Internet and stores it as packets in its buffer. Next, it sends the data packets to the users through both APs following a multiplexing scheme where the buffer service rate is divided according to a allocation ratio $\delta \in[0,1]$. Thus, Bob and Eve are assumed to be equipped with an RF front-end and a photo-diode (PD) and have multihoming capabilities, i.e., they can receive data from both APs simultaneously. Furthermore, the VLC and RF coverage areas are assumed to overlap (with the latter being larger than the former) [10].

\section{A. RF Channel Model}

Both RF links are assumed to undergo independent Rician block fading to justify the possible Line-of-Sight (LoS) component in an indoor scenario [11]. For notation simplicity, we consider Bob and Eve as B and E throughout the analysis. Thus, $h_{\mathrm{B}}^{\mathrm{rf}}$ and $h_{\mathrm{E}}^{\mathrm{rf}}$ denotes the channel coefficients between $\mathrm{S} \rightarrow \mathrm{B}$ and $\mathrm{S} \rightarrow \mathrm{E}$, respectively, which are modeled with shape factor $K_{B}$ and $K_{E}$ and scale parameter equal to the average channel gain of the corresponding link, $\Omega_{i}=\mathbb{E}\left\{\left|\left(h_{i}^{\text {rf }}\right)^{2}\right|\right\}$, with $i \in\{\mathrm{B}, \mathrm{E}\}$. Therefore, the received signal at both receivers are expressed as

$$
y_{i}^{\mathrm{rf}}(t)=\sqrt{P^{\mathrm{rf}}} s^{\mathrm{rf}}(t) h_{i}^{\mathrm{rf}}(t)+n_{i}^{\mathrm{rf}}(t) .
$$

where $P^{\mathrm{rf}}$ is the total transmit power of the RF AP and $s^{\mathrm{rf}}(t)$ is the transmitted signal from S. $n_{i}^{\mathrm{rf}}(t)$ is the noise component, modeled as additive white Gaussian noise (AWGN) with average power $N_{0}$. Moreover, we assume that the information signal $s^{\mathrm{rf}}(t)$ has mean power normalised to unity, that is, $\mathbb{E}\left\{\left|\mathrm{s}^{\mathrm{rf}}(t)\right|^{2}\right\}=1$. For simplicity, consider $g_{i}^{\mathrm{rf}} \triangleq\left|h_{i}^{\mathrm{rf}}\right|^{2}$ for $i \in\{\mathrm{B}, \mathrm{E}\}$ as the channel gains. Thus, $\gamma_{i}^{\mathrm{rf}}=\gamma^{\mathrm{rf}} g_{i}^{\mathrm{rf}}$, with $i \in\{\mathrm{B}, \mathrm{E}\}$, denotes the signal-to-noise ratio (SNR) received at Bob and Eve, where $\gamma^{\mathrm{rf}}=\mathrm{P}^{\mathrm{rf}} / N_{0}$ is the total transmit system SNR with respect to the RF transmitter.

\section{B. VLC Channel Model}

For VLC, it is assumed to employ intensity modulation/direct detection (IM/DD). Thus, the emitted light intensity varies according to the transmitted signal. Hence, the transmitted signal must be nonnegative, that is, $s^{\mathrm{vlc}} \in$ $\mathbb{R}^{+}$[12]. Furthermore, the VLC front-end at Bob and Eve are equipped with PDs, responsible to generate an electrical current proportional to the collected light intensity. Moreover, since in typical indoor scenarios the majority of the collected energy at the PDs comes from the LoS components, we assume that the VLC channel is flat with a dominant LoS component, and the channel gain does not vary during the data transmission as long as the receivers are stationary [13]. Therefore, for a time instant $t$, the received signal at the PDs of both users is given as follows

$$
y_{i}^{\mathrm{vlc}}(t)=s^{\mathrm{vlc}}(t) h_{i}^{\mathrm{vlc}}+n_{i}^{\mathrm{vlc}}(t)
$$

where $h^{\text {vlc }} \in \mathbb{R}^{+}$is the optical channel gain, $n_{i}^{\text {vlc }}(t)$ is the noise component. Moreover, due to safety concerns, the average value of $s^{\text {vlc }}$ is upper bounded as $\mathbb{E}\left\{s^{\text {vlc }}\right\}=P^{\text {vlc }}$. Thus, the channel gain at a given distance between the AP and the users are expressed as [14]

$$
h_{i}^{\mathrm{vlc}}=\frac{(m+1) A D(\psi) r^{2} d v^{m+1}}{2 \pi \sin ^{2}\left(\psi_{C}\right) d_{i}^{m+3}},
$$

where $d_{i}$, with $i \in\{\mathrm{B}, \mathrm{E}\}$, is the distance from the transmitter to the receiver, $d v$ is the vertical distance between the AP and the floor, $A$ denotes the area of the PD, $\psi$ and $\psi_{C}$ are, respectively, the angle of incidence with respect to the normal axis to the receiver plane, and the field of view (FOV) angle of the PD. $D(\psi)$ is the gain of the optical filter, and $r$ is the refractive index. $m=-1 / \log _{2}\left(\cos \left(\phi_{1 / 2}\right)\right)$ represents the order of Lambertian emission, where $\phi_{1 / 2}$ is the LED half intensity viewing angle. Following the optical-to-electrical conversion, the SNR at the VLC receivers can be defined as [15]

$$
\gamma_{i}^{\mathrm{vlc}}=\frac{\left(\rho P^{\mathrm{vlc}} h_{i}^{\mathrm{vlc}}\right)^{2}}{k^{2} N_{0}},
$$

where $\rho$ is the optical-to-electrical conversion efficiency of the $\mathrm{PD}$, and $k$ is the ratio between the average optical power and the average electrical power of the transmitted signal. 


\section{Source Model}

We assume that the data to be transmitted is first stored in a buffer following a two-state discrete-time Markov process with $\mathrm{ON}$ and $\mathrm{OFF}$ states in each time frame, i.e., when the source is in the ON state, the data arrives at the transmitter with a constant data arrival rate of $\lambda$ bits per frame. On the OFF state, no data is arriving at the transmitter buffer. Therefore, we define the transition probability from the ON state to the OFF state as $1-\alpha$, and the transition from the OFF state to the ON state as $1-\beta$. The probabilities of staying in the $\mathrm{ON}$ and $\mathrm{OFF}$ state are denoted by $\alpha$ and $\beta$, respectively. Hence, the transition matrix can be written as

$$
J=\left[\begin{array}{cc}
\alpha & 1-\alpha \\
1-\beta & \beta
\end{array}\right]
$$

Let us define the steady-state probabilities of the data arrival process being in the $\mathrm{ON}$ and OFF states as $p_{\mathrm{ON}}$ and $p_{\mathrm{OFF}}$, respectively, so that $p_{\mathrm{ON}}+p_{\mathrm{OFF}}=1$ and

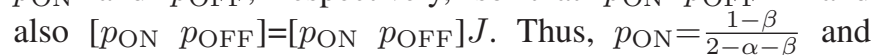
$p_{\mathrm{OFF}}=\frac{1-\alpha}{2-\alpha-\beta}$. Hence, the average data arrival rate at the transmitter buffer is

$$
r_{a v g}=\lambda p_{\mathrm{ON}}=\frac{\lambda(1-\beta)}{2-\alpha-\beta} .
$$

which is equal to the average service rate when the queue is in steady state [16].

\section{Performance AnAlysis}

In this section, an analytical expression for the effective capacity of the hybrid RF/VLC system studied is presented. To this end, we begin by revising the definition of the secrecy capacity $C_{s}$ as the maximum transmission rate achievable for a secure communication, given by the difference between the capacity of the legitimate and wiretap channels. Thus the secrecy capacity for the RF and VLC channels are given, respectively, by [17], [18]:

$$
\begin{gathered}
C_{s}^{\mathrm{rf}}=\left[C_{\mathrm{B}}^{\mathrm{rf}}-C_{\mathrm{E}}^{\mathrm{rf}}\right]^{+}=\left[\log _{2}\left(\frac{1+\gamma_{\mathrm{B}}^{\mathrm{rf}}}{1+\gamma_{\mathrm{E}}^{\mathrm{rf}}}\right)\right]^{+}, \\
C_{s}^{\mathrm{vlc}}=\frac{1}{2}\left[C_{\mathrm{B}}^{\mathrm{vlc}}-C_{\mathrm{E}}^{\mathrm{vlc}}\right]^{+}=\frac{1}{2}\left[\ln \left(\frac{1+c^{2} \gamma_{\mathrm{B}}^{\mathrm{vlc}}}{1+c^{2} \gamma_{\mathrm{E}}^{\mathrm{vlc}}}\right)\right]^{+},
\end{gathered}
$$

where $c$ is some constant. For instance, when $s^{\text {vlc }}$ is exponentially distributed, $c$ is set as $\sqrt{e / 2 \pi}$ [19]. Moreover, the transmitter is assumed to perfectly knows the channel state information (CSI) of the links with Bob and Eve in each frame. The estimation of the channels are performed at the receivers and forwarded to the transmitter in a delay-free and error-free feedback channel [20]. Besides, we further assume that the maximal achievable secrecy rate for the RF and VLC channel is set as the secrecy capacity, given in bits per frame by $\mathcal{R}_{\mathrm{s}}^{i} \approx C_{\mathrm{s}}^{i}$ with $i \in\{\mathrm{rf}, \mathrm{vlc}\}$.

\section{A. Queueing Constraints}

Since the data is first stored in a buffer, we assume that statistic constraints are imposed on the queue length. Hence, we consider that the buffer violation/overflow probability satisfies [21]

$$
\theta=-\lim _{q \rightarrow \infty} \frac{\ln \operatorname{Pr}\{Q \geq q\}}{q},
$$

where $Q$ is the stationary queue length, $\theta>0$ is the decay rate of the tail distribution of the queue length and $q$ is the buffer overflow threshold. For a large $q$, (9) can be approximated as $\operatorname{Pr}\{Q \geq q\} \approx e^{-\theta q}$. Thus, the buffer overflow probability decays exponentially with the rate set to the QoS exponent $\theta$.

Next, we define the effective bandwidth and the effective capacity, necessary to formulate the maximum average arrival rate, one of the main performance metrics of the paper.

Definition 1.: The effective bandwidth characterizes the minimum constant transmission rate required to satisfy (9). Let $\{a(k), k=1,2, \ldots\}$ be a sequence of non-negative random variables, describing the random arrival rates. Let also the time-accumulated arrival process be denoted by $A(t)=\sum_{k=1}^{t} a(k)$. Then, the effective bandwidth is given by the asymptotic logarithmic moment generating function of $A(t)[22]$

$$
a^{*}(\theta)=\lim _{t \rightarrow \infty} \frac{1}{\theta t} \ln \mathbb{E}\left\{e^{\theta A(t)}\right\} .
$$

More specifically, since we consider a discrete-time Markov source, given the transition matrix in (5), the effective bandwidth of the transmission buffer can be derived as [21]

$$
a_{s}^{*}(\theta)=\frac{1}{\theta} \ln \left(\frac{\beta+\alpha e^{\lambda \theta}+\sqrt{\left(\beta+\alpha e^{\lambda \theta}\right)^{2}-4(\beta+\alpha-1) e^{\lambda \theta}}}{2}\right) \text {. }
$$

Definition 2.: The effective capacity identifies the maximum constant arrival rate supported by a given time-varying service process while satisfying (9). Let $\{v(k), k=1,2, \ldots\}$ denote the discrete-time stationary and ergodic stochastic service process and $S(t)=\sum_{k=1}^{t} v(k)$ be the time-accumulated service process. Then, the effective capacity is given by [23]

$$
C(\theta)=-\lim _{t \rightarrow \infty} \frac{1}{\theta t} \ln \mathbb{E}\left\{e^{-\theta S(t)}\right\} .
$$

As we assume that both links send data simultaneously according to $\delta$, the effective capacity can be written as

$$
\begin{aligned}
C(\theta) & =-\frac{1}{\theta} \ln \mathbb{E}\left\{e^{-\theta\left(\delta \mathcal{R}_{\mathrm{s}}^{\mathrm{rf}}+(1-\delta) \mathcal{R}_{\mathrm{s}}^{\mathrm{vlc}}\right)}\right\} \\
& =-\frac{1}{\theta}\left(\ln \mathbb{E}\left\{e^{-\theta\left(\delta \mathcal{R}_{\mathrm{s}}^{\mathrm{rf}}\right)}\right\}\right)+(1-\delta) \mathcal{R}_{\mathrm{s}}^{\mathrm{vlc}}
\end{aligned}
$$

Proposition 1. An integral form for the Effective Capacity of a hybrid RF-VLC system with statistical constraints in the presence of an eavesdropper is given by

$$
C(\theta)=-\frac{\ln \omega_{1}+\omega_{2}}{\theta}+(1-\delta) \frac{1}{2}\left[\ln \left(\frac{1+c^{2} \gamma_{\mathrm{B}}^{\mathrm{vlc}}}{1+c^{2} \gamma_{\mathrm{E}}^{\mathrm{vlc}}}\right)\right]^{+}
$$


where,

$$
\begin{aligned}
\omega_{1} & =\int_{0}^{\infty} \int_{\mathrm{g}_{\mathrm{E}}^{\mathrm{rf}}}^{\infty} e^{-\delta \theta \log _{2}\left(\frac{1+\gamma^{\mathrm{rf}} g_{\mathrm{B}}^{\mathrm{rf}}}{1+\gamma^{\mathrm{rf}} g_{\mathrm{E}}^{\mathrm{rf}}}\right)} f_{g_{B}^{\mathrm{rf}}}(x) f_{g_{\mathrm{E}}^{\mathrm{rf}}}(y) d x d y \\
\omega_{2} & =\sum_{l=0}^{\infty} \sum_{n=0}^{\infty}\left(-{ }_{2} F_{1}\left(n+1, l+n+2 ; n+2 ; \frac{-\left(K_{\mathrm{E}}+1\right) d_{\mathrm{B}}^{-\phi} d_{\mathrm{E}}^{\phi}}{K_{\mathrm{B}}+1}\right)\right. \\
& \left.\times \frac{\left(K_{\mathrm{E}}+1\right)^{n+1} d_{\mathrm{E}}^{(n+1) \phi} \Gamma(l+n+2)}{\left(K_{\mathrm{B}}+1\right)^{n+1}}+\Gamma(l+1) d_{\mathrm{B}}^{n \phi+\phi}\right) \\
& \times \frac{e^{-K_{\mathrm{B}}-K_{\mathrm{E}}} K_{\mathrm{B}}^{l} K_{\mathrm{E}}^{n} \mathrm{db}(-n-1) \phi}{\Gamma(l+1)^{2} \Gamma(n+1) \Gamma(n+2)} .
\end{aligned}
$$

Proof. The proof is provided in Appendix A.

\section{B. Asymptotic Effective Capacity}

To gain a better insight into the secrecy behavior of the investigated system, an asymptotic expression for the effective capacity is determined in the following proposition.

Proposition 2. An asymptotic expression for the effective capacity of a hybrid RF-VLC system with statistical constraints in the presence of an eavesdropper is given by

$$
C(\theta)^{\infty}=-\frac{\ln \omega^{\infty}}{\theta}+(1-\delta) \frac{1}{2}\left[\ln \left(\frac{1+c^{2} \gamma_{\mathrm{B}}^{\mathrm{vlc}}}{1+c^{2} \gamma_{\mathrm{E}}^{\mathrm{vlc}}}\right)\right]^{+},
$$

where $\omega^{\infty}$ is given by (18) at the top of the next page.

Proof. By considering the high SNR regime (i.e., as $\gamma^{\mathrm{rf}} \rightarrow \infty$ ) into (7), we have that $1 / \gamma^{\mathrm{rf}} \rightarrow 0$ as $P^{\mathrm{rf}}$ becomes larger in the high SNR regime. Thus, after some simplifications, the asymptotic expression for $C(\theta)$ can be obtained as in (18).

\section{Average Arrival Rate}

We aim to identify the maximum average arrival rate supported while satisfying the statistical QoS limitations given in (9). On this matter, (9) is satisfied if the effective bandwidth of the arrival process is equal to the effective capacity of the service process, that is, $a^{*}(\theta)=C(\theta)$. Hence, for the two-state (ON/OFF) discrete Markov source, the maximum average arrival rate is expressed as [16]

$$
r_{\mathrm{avg}}^{*}=\frac{p_{\mathrm{ON}}}{\theta} \ln \left(\frac{e^{2 \theta C(\theta)}-\beta e^{\theta C(\theta)}}{1-\alpha-\beta+\alpha e^{\theta C(\theta)}}\right) .
$$

\section{NUMERICAL RESULTS AND DISCUSSIONS}

In this section, the analytical expressions derived in Section III are evaluated and validated via Monte Carlo simulations. For the presented results, we have considered $l$ and $n$ in (18) equal to 30 . The probability of the buffer staying in the ON and OFF state are assumed $\alpha=0.5$ and $\beta=0.5$, respectively. We have also considered that the users positions are estimated based on the LED half intensity viewing angle, i.e. $d_{i}=d v / \cos \left(\phi_{1 / 2}\right)$ with $i \in\{\mathrm{B}, \mathrm{E}\}$. Moreover, the average channel gains of the RF links are assumed to
TABLE I: Simulation Parameters

\begin{tabular}{l|c}
\hline \multicolumn{2}{c}{ RF subsystem } \\
\hline Transmit SNR, $\gamma^{\text {rf }}$ & $10 \mathrm{~dB}$ \\
Rician factor, K & $10 \mathrm{~dB}$ \\
Path Loss Exponent, $\phi$ & 1.8 \\
\hline \multicolumn{2}{c}{ VLC subsystem } \\
\hline Vertical distance, $d v$ & $2.5 \mathrm{~m}$ \\
PD field of view (FOV), $\psi_{C}$ & $90^{\circ}$ \\
Average emitted power, $P^{\mathrm{vlc}}$ & $9 \mathrm{~W}$ \\
PD physical area, A & $1 \mathrm{~cm}^{2}$ \\
PD responsivity, $\rho$ & $0.53 \mathrm{~A} / \mathrm{W}$ \\
Refractive index, $\mathrm{r}$ & 1.5 \\
Optical filter gain, $D(\psi)$ & 1 \\
LED half intensity viewing angle, $\phi_{1 / 2}$ & $60^{\circ}$ \\
Noise power spectral density, $N^{\mathrm{vlc}}$ & $10^{-21} \mathrm{~A}^{2} / \mathrm{Hz}$ \\
Elect./opt. power conversion, $\mathrm{k}$ & 3 \\
Constant c & $\sqrt{e / 2 \pi}$ \\
\hline
\end{tabular}

be determined by the path loss, i.e., $\Omega_{i}=d_{i}^{-\varphi}, i \in\{\mathrm{B}, \mathrm{E}\}$. Furthermore, unless otherwise specified, Table I summarises some of the considered parameters.

Fig. 2 illustrates the $r_{\text {avg }}^{*}$ as a function of the distance ratio between the nodes, $d_{B} / d_{E}$ for two values of allocation ratio $\delta=0.2,0.8$ and $\theta=1,5,10$. Moreover, we fixed the position of Eve at edge of the VLC AP coverage. It can be observed that our analytical expression perfectly matches the simulation results. Note that, as expected, larger values of $\theta$ result in stricter QoS constraints which entail fewer data arriving at the buffer, independently of the allocation ratio considered. Still, the influence of the QoS exponent on the system is more pronounced when the amount of data transmitted via RF is higher, which is explained by the stochastic nature of the RF channel, that prevents the RF link from supporting data arrival rates at the transmitter buffer when the QoS constraints are stricter [20]. Furthermore, note that, as expected, when Bob is closer to the source, it is preferable to allocate more data to the VLC since it can transmit at higher data rates with secrecy. However, as Bob approaches Eve's location, there is a trade-off in performance, and it is preferable to allocate more data to the RF AP.

Fig. 3 shows the $r_{\text {avg }}$ vs. the QoS exponent, $\theta$, for different allocation ratios $\delta=0.2,0.5,0.8$ and $\gamma^{\text {rf }}=10,20 \mathrm{~dB}$. We further assume Bob located beneath the source and Eve positioned at the edge of the VLC coverage. As observed in the previous figure, increasing the QoS exponent reduces the amount of data arriving at the buffer independently of the allocation ratio. However, since the VLC link is more robust to QoS restrictions, the scenarios with $\delta=0.2$ present a smaller decrease in performance. On the other hand, note that as more data is allocated to the RF link, its stochastic nature is beneficial to the system performance when looser queuing constraints are applied. Also, observe that for the considered position of the nodes, increasing the amount of power available for transmission at the RF channel can impact the performance even with smaller values of $\delta$.

To complement the results, Figs. 4 and 5 illustrate $C(\theta)$ versus the transmit SNR and the allocation ratio, $\delta$, respec- 


$$
\begin{aligned}
\omega^{\infty} & =\sum_{l=0}^{\infty} \sum_{n=0}^{\infty} \frac{e^{-K_{\mathrm{B}}-K_{\mathrm{E}}} K_{\mathrm{B}}^{l} K_{\mathrm{E}}^{n}}{\Gamma(l+1)^{2} \Gamma(n+1)^{2}}\left(\Gamma(l+1) \Gamma(n+1)-\left(K_{\mathrm{B}}+1\right)^{-n-1}\left(K_{\mathrm{E}}+1\right)^{n+1} d_{\mathrm{B}}^{(-n-1) \phi} d_{\mathrm{E}}^{(n+1) \phi} \Gamma(l+n+2)\right. \\
& \left.\times\left(\frac{{ }_{2} F_{1}\left(n+1, l+n+2 ; n+2 ;-\frac{d_{\mathrm{B}}^{-\phi} d_{\mathrm{E}}^{\phi}\left(K_{\mathrm{E}}+1\right)}{K_{\mathrm{B}}+1}\right)}{n+1}-\frac{\log (2){ }_{2} F_{1}\left(l+n+2, n+\frac{\delta \theta}{\log (2)}+1 ; n+\frac{\delta \theta}{\log (2)}+2 ;-\frac{d_{\mathrm{B}}^{-\phi} d_{\mathrm{E}}^{\phi}\left(K_{\mathrm{E}}+1\right)}{K_{\mathrm{B}}+1}\right)}{\delta \theta+n \log (2)+\log (2)}\right)\right)
\end{aligned}
$$

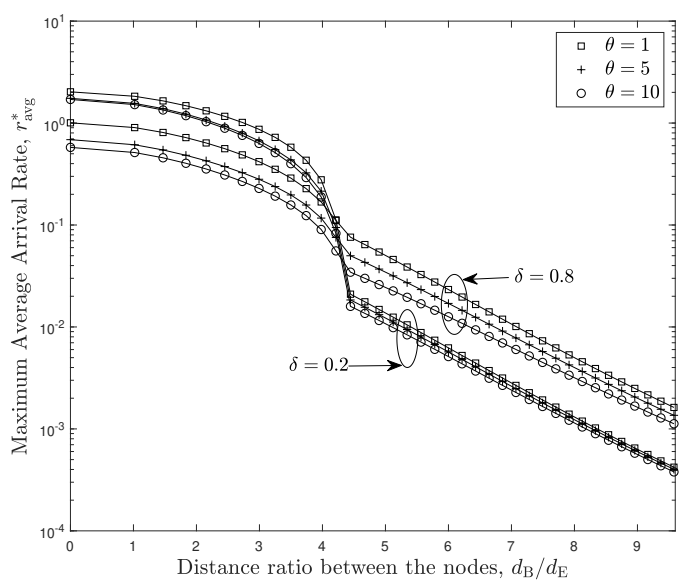

Fig. 2: Maximum Average Arrival Rate, $r_{\text {avg }}^{*}$ vs Distance ratio between nodes, $d_{B} / d_{E}$ for different allocation ratios $\delta=0.2,0.8$ and $\theta=1,5,10$. Solid line represents the analytical expression; markers represents the simulation results.

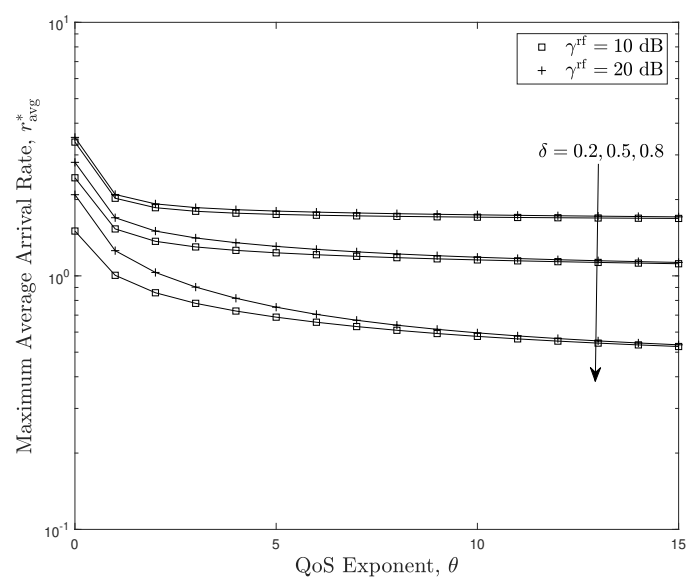

Fig. 3: Maximum Average Arrival Rate, $r_{\text {avg }}$ vs QoS Exponent, $\theta$ for different allocation ratios $\delta=0.2,0.5,0.8$ and $\gamma^{\mathrm{rf}}=10,20 \mathrm{~dB}$. Solid line represents the analytical expression; markers represents the simulation results.

tively. Fig. 4 considers $\delta=0.2,0.5,0.8$ and $d_{\mathrm{B}} / d_{\mathrm{E}}=0.5,0.95$. From this figure, observe that our derived asymptotic expression in (18) is independent of $\gamma^{\mathrm{rf}}$ at high SNR, which indicates that $C(\theta)$ presents a saturation. Furthermore, note

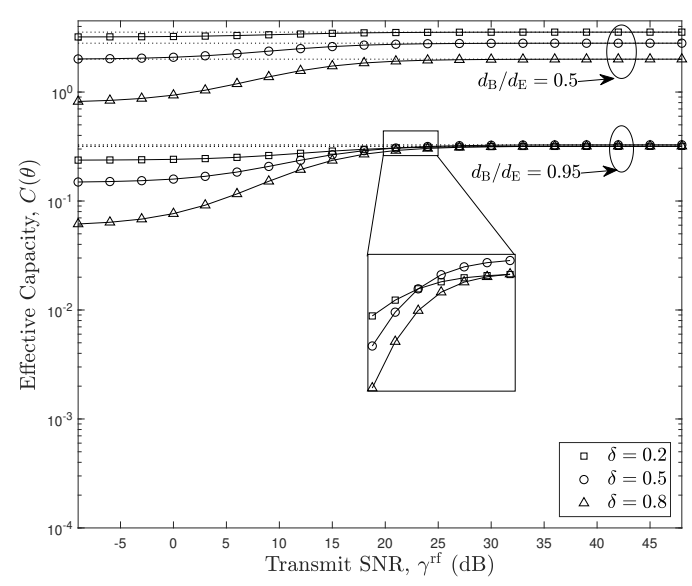

Fig. 4: Effective Capacity, $C(\theta)$ vs transmit SNR for different allocation ratios $\delta=0.2,0.5,0.8$ and $d_{B} / d_{E}=0.5,0.95$ with $\theta=1$. Solid and dashed line represents the analytical and asymptotic expressions, respectively; markers represents the simulation results.

that as Bob moves to the VLC coverage's edge, where Eve is located, the system's performance in terms of the transmit SNR is also independently of $\delta$. Besides, it can be seen that the transmit SNR of the RF channel can influence the point of the trade-off of allocation between the two channels.

In Fig. 5, the trade-off of allocation is examined. As expected, with Bob in the vicinity of $S$, to enhance performance, $\delta$ should be smaller, as it is preferable to send more data through the VLC link. On the other hand, as the distance between the nodes increases, smaller values of $\delta$ severely impact the system performance. This is explained by the VLC channel characteristics exhibited in (3), that is, the secrecy capacity of the VLC channel depends solemnly on the distance between the users and the AP. Therefore, the system can't guarantee a secure transmission relying on the VLC link if Eve is closer to the source than Bob. Which indicates the advantages of combining VLC and RF to guarantee secrecy.

\section{CONCLUSION}

This paper investigated the performance in terms of the effective capacity and the maximum average arrival rate of a hybrid RF/VLC system in the presence of an eavesdropper where the data is first stored in a buffer that operates under queuing constraints. An integral-form and an asymp- 


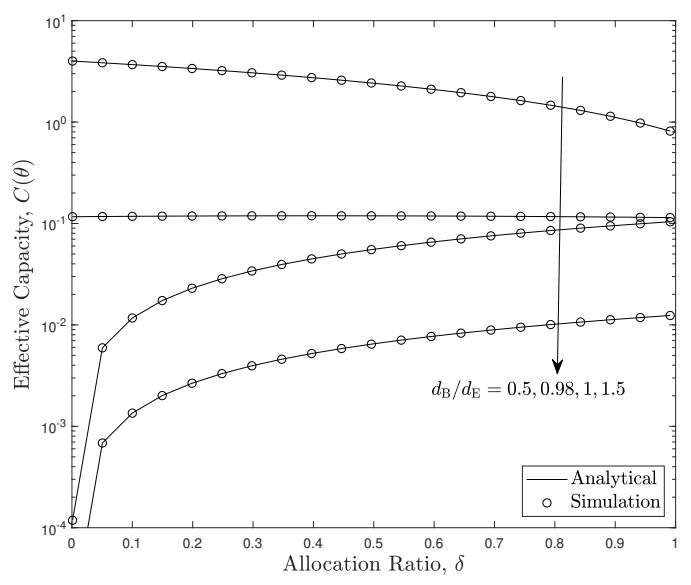

Fig. 5: Effective Capacity, $C(\theta)$ vs the allocation ratio, $\delta$ for different average distances between nodes, $d_{B} / d_{E}$, with $\theta=1$.

totic expression for the effective capacity were derived. Our analytical expressions were corroborated via Monte Carlo simulations. The results showed that the VLC channel is robust and can ensure a secure transmission to the legitimate receiver. However, since the VLC channel gain is based on the distance between the AP and the users and the coverage area is limited, a hybrid RF/VLC system can enhance the coverage and guarantee secure transmission of the confidential signal even under stricter QoS constraints.

\section{ACKNOWLEDGEMENT}

This work was partially supported by the Academy of Finland, 6Genesis Flagship under Grant 318927 and FAITH project under Grant 334280.

\section{APPENDIX A}

\section{ProOF OF PROPOSITION 1}

We start by rewritten $C_{S}^{\mathrm{rf}}$, previously defined in (7) as

$$
C_{S}^{\mathrm{rf}}= \begin{cases}0, & g_{b}^{\mathrm{rf}} \leq g_{e}^{\mathrm{rf}} \\ \log _{2}\left(\frac{1+\gamma^{\mathrm{rf}} g_{\mathrm{B}}^{\mathrm{rf}}}{1+\gamma^{\mathrm{rf}} g_{\mathrm{E}}^{\mathrm{rf}}}\right), & g_{b}^{\mathrm{rf}}>g_{e}^{\mathrm{rf}}\end{cases}
$$

thus, by knowing that $\mathbb{E}\left\{e^{-\theta \delta C_{S}^{\mathrm{rf}}}\right\}$ is given by

$$
\mathbb{E}\left\{e^{-\theta \delta C_{S}^{\mathrm{rf}}}\right\}=\int_{0}^{\infty} e^{-\theta \delta C_{S}^{\mathrm{rf}}} f_{g_{i}^{\mathrm{rf}}}\left(g_{i}^{\mathrm{rf}}\right) d g_{i}^{\mathrm{rf}}
$$

we can split (21) regarding the valid cases of (20) as $\omega_{1}$ in (15) and $\omega_{2}$ can be described as

$$
\omega_{2}=\int_{0}^{\infty} \int_{0}^{\mathrm{g}_{\mathrm{E}}^{\mathrm{rf}}} f_{g_{B}^{\mathrm{rf}}}(x) f_{g_{E}^{\mathrm{rf}}}(y) d x d y .
$$

next, given that the RF channel coefficients are assumed to undergo Rician fading, hence $g_{i}^{\text {rf }}$, with $i \in\{\mathrm{B}, \mathrm{E}\}$ follow a non-central chi-squared distribution. Also, considering the series expansion of the Bessel function as given in [9, Eq. 8.447.1] and by using [9, Eq. 3.351.1] and [9, Eq. 6.455.1], after some simplifications, (16) can be reached.

\section{REFERENCES}

[1] G. Wikström and et al., "Challenges and technologies for 6G," in 2020 2nd $6 G$ Wireless Summit (6G SUMMIT), 2020, pp. 1-5.

[2] M. S. Saud, I. Ahmed, T. Kumpuniemi, and M. Katz, "Reconfigurable optical-radio wireless networks: Meeting the most stringent requirements of future communication systems," Trans. on Emerging Telecommun. Technol., vol. 30, no. 2, p. e3562, 2019.

[3] N. Chi, Y. Zhou, Y. Wei, and F. Hu, "Visible light communication in 6g: Advances, challenges, and prospects," IEEE Veh. Technol. Magazine, vol. 15 , no. 4, pp. 93-102, 2020.

[4] D. A. Basnayaka and H. Haas, "Design and analysis of a hybrid radio frequency and visible light communication system," IEEE Trans. on Commun., vol. 65, no. 10, pp. 4334-4347, 2017.

[5] H. Chowdhury and M. Katz, "Cooperative data download on the move in indoor hybrid (radio-optical) wlan-vlc hotspot coverage," Trans. on Emerging Telecommmun. Technol., vol. 25, no. 6, pp. 666-677, 2014.

[6] D. P. M. Osorio, E. E. B. Olivo, H. Alves, and M. Latva-Aho, "Safeguarding mtc at the physical layer: Potentials and challenges," IEEE Access, vol. 8, pp. 101 437-101 447, 2020.

[7] M. F. Marzban, M. Kashef, M. Abdallah, and M. Khairy, "Beamforming and power allocation for physical-layer security in hybrid rf/vlc wireless networks," in 2017 13th International Wireless Communications and Mobile Computing Conference (IWCMC), 2017, pp. 258-263.

[8] J. Al-Khori, G. Nauryzbayev, M. M. Abdallah, and M. Hamdi, "Secrecy performance of decode-and-forward based hybrid rf/vlc relaying systems," IEEE Access, vol. 7, pp. 10 844-10 856, 2019.

[9] I. S. Gradshteyn and I. M. Ryzhik, Table of integrals, series, and products, 7th ed. Elsevier/Academic Press, Amsterdam, 2007.

[10] F. Wang, Z. Wang, C. Qian, L. Dai, and Z. Yang, "Efficient vertical handover scheme for heterogeneous vlc-rf systems," IEEE/OSA Journal of Opt. Commun. and Net., vol. 7, no. 12, pp. 1172-1180, 2015.

[11] M. Duarte, C. Dick, and A. Sabharwal, "Experiment-driven characterization of full-duplex wireless systems," IEEE Trans. on Wireless Commun., vol. 11, no. 12, pp. 4296-4307, 2012.

[12] J.-B. Wang and et al., "Tight bounds on channel capacity for dimmable visible light communications," Journal of Lightw. Technol., vol. 31, no. 23, pp. 3771-3779, 2013.

[13] T. Komine and M. Nakagawa, "Fundamental analysis for visible-light communication system using led lights," IEEE Trans. on Consumer Electronics, vol. 50, no. 1, pp. 100-107, 2004.

[14] J. R. Barry and et al., "Simulation of multipath impulse response for indoor wireless optical channels," IEEE Journal on Sel. Areas in Commun., vol. 11, no. 3, pp. 367-379, 1993.

[15] Y. Wang and H. Haas, "Dynamic load balancing with handover in hybrid li-fi and wi-fi networks," Journal of Lightw. Technol., vol. 33, no. 22, pp. 4671-4682, 2015.

[16] C.-S. Chang, Performance Guarantees in Communication Networks. Berlin, Heidelberg: Springer-Verlag, 2000.

[17] S. Leung-Yan-Cheong and M. Hellman, "The gaussian wire-tap channel," IEEE Trans. on Inf. Theory, vol. 24, no. 4, pp. 451-456, 1978.

[18] A. Chaaban, Z. Rezki, and M. Alouini, "Fundamental limits of parallel optical wireless channels: Capacity results and outage formulation," IEEE Trans. on Commun., vol. 65, no. 1, pp. 296-311, 2017.

[19] A. Lapidoth, S. M. Moser, and M. A. Wigger, "On the capacity of freespace optical intensity channels," IEEE Trans. on Inf. Theory, vol. 55, no. 10, pp. 4449-4461, 2009.

[20] M. Hammouda and et al., "Link selection in hybrid RF/VLC systems under statistical queueing constraints," IEEE Trans. on Wireless Commun., vol. 17, no. 4, pp. 2738-2754, 2018

[21] M. Ozmen and M. C. Gursoy, "Wireless throughput and energy efficiency with random arrivals and statistical queuing constraints," IEEE Trans. on Inf. Theory, vol. 62, no. 3, pp. 1375-1395, March 2016.

[22] Cheng-Shang Chang, "Stability, queue length, and delay of deterministic and stochastic queueing networks," IEEE Trans. on Automatic Control, vol. 39, no. 5, pp. 913-931, 1994.

[23] Dapeng Wu and R. Negi, "Effective capacity: a wireless link model for support of quality of service," IEEE Trans. on Wireless Commun., vol. 2 , no. 4, pp. 630-643, 2003. 